(c) American Dairy Science Association, 2005.

\title{
Thermal and Structural Behavior of Anhydrous Milk Fat. 3. Influence of Cooling Rate
}

\author{
C. Lopez, ${ }^{*, 1}$ P. Lesieur, ${ }^{2,3}$ C. Bourgaux, ${ }^{2}$ and M. Ollivon ${ }^{1}$ \\ ${ }^{1}$ Equipe Physico-Chimie des Systèmes Polyphasés, UMR 8612 du CNRS, \\ 5 rue J.B. Clément 92296, Châtenay-Malabry, France \\ 'Laboratoire pour l'Utilisation du Rayonnement Electromagnétique, \\ Bât. 209D Université Paris-sud, 91898 Orsay, France \\ ${ }^{3}$ Physico-Chimie des Colloïdes, Université Henri Poincaré, UMR7565 BP 239, \\ 54506 Vandoeuvre-lès-Nancy, France
}

\section{ABSTRACT}

The crystallization behavior of anhydrous milk fat has been examined with a new instrument coupling timeresolved synchrotron x-ray diffraction as a function of temperature (XRDT) at both small and wide angles and high-sensitivity differential scanning calorimetry. Crystallizations were monitored at cooling rates of 3 and $1^{\circ} \mathrm{C} /$ min from 60 to $-10^{\circ} \mathrm{C}$ to determine the triacylglycerol organizations formed. Simultaneous thermal analysis permitted the correlation of the formation/melting of the different crystalline species monitored by XRDT to the thermal events recorded by differential scanning calorimetry. At intermediate cooling rates, milk fat triacylglycerols sequentially crystallize in 3 different lamellar structures with double-chain length of 46 and $38.5 \AA$ and a triple-chain length of $72 \AA$ stackings of $\alpha$ type, which are correlated to 2 exothermic peaks at 17.2 and $13.7^{\circ} \mathrm{C}$, respectively. A time-dependent slow sub- $\alpha \leftrightarrow \alpha$ reversible transition is observed at $-10^{\circ} \mathrm{C}$. Subsequent heating at $2^{\circ} \mathrm{C} / \mathrm{min}$ has shown numerous structural rearrangements of the $\alpha$ varieties into a single $\beta^{\prime}$ form before final melting. This polymorphic evolution on heating, as well as the final melting point observed $\left(\sim 39^{\circ} \mathrm{C}\right)$, confirmed that cooling at $3^{\circ} \mathrm{C} / \mathrm{min}$ leads to the formation of crystalline varieties that are not at equilibrium. An overall comparison of the thermal and structural properties of the crystalline species formed as a function of the cooling rate and stabilization time is presented. The influence on crystal size of the cooling rates applied in situ using temperature-controlled polarized microscopy is also determined for comparison.

(Key words: triacylglycerol, polymorphism, differential scanning calorimetry, x-ray diffraction)

Received April 9, 2004.

Accepted July 15, 2004

Corresponding author: C. Lopez; e-mail: Christelle.Lopez@rennes. inra.fr.

*Present address: UMR 1253, Science et Technologie du Lait et de l'Oeuf, INRA-Agrocampus 65, rue de Saint Brieuc 35042 Rennes, France.
Abbreviation key: AMF = anhydrous milk fat, DSC = differential scanning calorimetry, $\mathbf{2 L}=$ bi-layered stacking, $\mathbf{3 L}=$ tri-layered stacking, $\mathbf{S A X D}=$ small-angle XRD, $\mathbf{T}=$ temperature, $\mathbf{T G}=$ triacylglycerol, $\mathbf{W A X D}=$ wideangle XRD, XRD $=$ x-ray diffraction, $\mathbf{X R D T}=\mathrm{XRD}$ as a function of $\mathrm{T}$.

\section{INTRODUCTION}

Fat, one of the major constituents of milk, contributed to the physical properties of dairy products, especially of low water content. It is also an important ingredient in the production of many bakery and confectionary foods. The functional properties of milk fat are strongly related to its composition and to the amount and the type of crystals formed at the temperature (T) of the application. Indeed, milk fat crystallization is important for technological applications of high-fat content products. Milk fat is mainly composed of triacylglycerols (TG) that represent about $97 \%$ of the total fat (Christie, 1995). The large number of TG, which vary in chain length and degree of saturation, is responsible for the broad melting range of milk fat that spans from about -40 to $40^{\circ} \mathrm{C}$. Moreover, the polymorphism associated with each TG increases the complexity of the structural and thermal behaviors of milk fat.

The different polymorphic forms observed for TG were detailed by different authors (Small, 1986; Hagemann, 1988; Ollivon and Perron, 1992). The 3 major polymorphic forms identified are $\alpha, \beta^{\prime}$, and $\beta$ in increasing order of stability. These polymorphic forms can be identified by x-ray diffraction (XRD).

Mulder (1953) was likely the first to report a doublemelting behavior for milk fat and to ascribe this behavior to the occurrence of polymorphism. Some studies have focused on the differences between the final diffraction pattern obtained after rapid and slow cooling (deMan, 1961; Woodrow and deMan, 1968). The final diffraction patterns recorded for milk fat showed mainly the $\beta^{\prime}$ form with traces of $\beta$ form in the samples that were slowly cooled. Timms (1979) found a very weak intensity of the 
peak characteristic of $\beta$ form in slowly cooled milk fat. Timms (1980) stated that partial transformation of milk fat crystals to the $\beta$ form can only occur if a considerable amount of milk fat is still liquid. van Beresteyn (1972) reported the formation of $\alpha$ and $\beta^{\prime}$ forms. After slow cooling at $0.1^{\circ} \mathrm{C} / \mathrm{min}$ to $28^{\circ} \mathrm{C}$ and after storage of the sample at $28^{\circ} \mathrm{C}$ for $24 \mathrm{~h}$, Schaap et al. (1975) also observed both the $\alpha$ and $\beta^{\prime}$ forms.

In all of these studies, the researchers have made a distinction between slow and rapid cooling but often without mentioning the exact cooling rates. However, as a consequence of polymorphism, fat behavior is, to a large extent, determined by the rate of cooling. Moreover, the polymorphic forms were often examined after considerable stabilization periods because of lengthy exposure times using XRD without taking into account the evolution of the samples.

Recently, access to x-ray high-flux sources (synchrotron) has permitted the study of the polymorphism and phase transitions displayed by complex TG mixtures as a function of $\mathrm{T}$ and the study of these data in comparison with differential scanning calorimetry (DSC) recordings (Lavigne, 1995; Keller et al., 1996; Loisel et al., 1998; ten Grotenhuis et al., 1999). ten Grotenhuis et al. (1999) studied polymorphism of milk fat by DSC and real-time $\mathrm{x}$-ray powder diffraction at small angles, from 70 to $-65^{\circ} \mathrm{C}$, at different cooling rates varying from 0.5 to $20^{\circ} \mathrm{C} /$ $\min$. They observed the formation of the sub- $\alpha, \alpha$, and $\beta^{\prime}$ polymorphic forms as a function of the cooling rate $|\mathrm{dT} / \mathrm{dt}|$. For $|\mathrm{dT} / \mathrm{dt}| \geq 2.5^{\circ} \mathrm{C} / \mathrm{min}$, the least stable sub- $\alpha$ form was recorded for $\mathrm{T} \leq-8^{\circ} \mathrm{C}$, together with $\alpha$ crystals. For $|\mathrm{dT} / \mathrm{dt}|=1.67^{\circ} \mathrm{C} / \mathrm{min}$, only $\alpha$ crystals were formed. For $|\mathrm{dT} / \mathrm{dt}| \leq 1^{\circ} \mathrm{C} / \mathrm{min}$, they observed the formation of $\beta^{\prime}$ forms together with a small amount of $\alpha$ form. The $\beta^{\prime}$ form was the most stable form observed in their study. Lavigne (1995) studied the polymorphism of milk fat and its fractions by XRD as a function of T (XRDT), at both small and wide angles (SAXD and WAXD, respectively), to characterize the longitudinal stacking and the lateral packing of the TG molecules within the lamellar structures, respectively. The structural data were compared with DSC recordings obtained in similar conditions.

Authors who studied polymorphism of milk fat by DSC agree with the existence of 3 endotherms recorded on heating (Timms, 1980; Marangoni and Lencki, 1998). These 3 melting peaks were interpreted in terms of fractions of milk fat. These fractions were called low melting point, medium melting point, and high melting point fractions. Lavigne (1995) identified the main TG of each fraction and related them to the thermal and structural properties of the whole milk fat and its fractions.

In previous papers, we studied the formation of the most unstable crystalline structures formed by anhy- drous milk fat (AMF) TG after rapid quenching from the melt to $-8^{\circ} \mathrm{C}$ (Lopez et al., 2001b) and the crystalline species formed at very slow cooling rate (Lopez et al., 2001c) as well as their evolutions on heating. To characterize the structural behavior of milk fat at intermediate cooling rates, the crystallization behavior of AMF is studied here at -3 and $-1^{\circ} \mathrm{C} / \mathrm{min}$. The evolution of the crystalline structures formed during a subsequent heating at $2^{\circ} \mathrm{C} / \mathrm{min}$ is also considered as in the preceding papers of the series. The same thermal treatments were applied in parallel to creams to determine the influence of fat globule interface onto TG crystallization (Lopez et al., 2001a). To avoid ice formation, the cooling was limited to $-8^{\circ} \mathrm{C}$ in cream samples. Then, similar conditions, cooling to $-10^{\circ} \mathrm{C}$, are applied in this study to allow comparison of thermal and structural behavior in bulk and dispersed systems. Finally, the influence of cooling rate on the thermal and structural behavior of AMF is analyzed.

\section{MATERIALS AND METHODS}

\section{AMF}

Anhydrous milk fat was extracted from creams (fat content $=40 \% \mathrm{wt} / \mathrm{wt}$ ) studied in Lopez et al. (2001a). The creams, obtained from industrial skimming of fresh whole milks collected in Brittany in July 2000, were purchased by the dairy plant (Laiterie du Val d'Ancenis, Ancenis, France). The procedure of extraction of AMF from cream is the following: $8 \mathrm{~mL}$ of isopropanol (Carlo Erba Reagenti, Val de Rueil, France) was added to $10 \mathrm{~g}$ of cream, the mixture was vortexed, then $12 \mathrm{~mL}$ of hexane (Carlo Erba Reagenti) was added. The resulting mixture was vortexed again and centrifuged for 5 min at $500 \times$ $g$. The upper organic phase was separated, and added to a second organic phase obtained by a second extraction of the lower phase using $12 \mathrm{~mL}$ of hexane. The pooled fractions were solvent-evaporated under vacuum until constant weight (hexane + isopropanol content is expected to be $<1 \mathrm{ppm}$ ).

\section{XRDT/DSC Measurements}

Coupled, time-resolved XRDT, and high-sensitivity DSC experiments carried out in the same apparatus from the same sample were conducted on D22 bench of the high-energy synchrotron of LURE (Laboratoire pour l'Utilisation du Rayonnement Electromagnétique). The setup has been described previously (Lopez et al., 2001b). Briefly, D22 bench is equipped with 2 linear detectors that allow recording of simultaneous SAXD and WAXD data with sample-to-detector distances of 30 and 177 $\mathrm{cm}$, respectively. The XRDT data and DSC measurements were synchronously collected vs. time by a single microcomputer. Crystalline $\beta$ form of high purity 
tristearin, synthetized and purified in the laboratory (Lavigne et al., 1993), was used as reference for wideangle channel to scattering vector calibration of the detector. Silver behenate was used as reference to calibrate the XRD data collected at small angles (Blanton et al., 2000).

Melted AMF samples $(\sim 30 \mu \mathrm{L})$ were loaded in thin Lindeman glass capillaries (GLAS; Muller, Berlin, Germany) especially designed for XRD. The glass capillaries allow minimum attenuation of the beam and parasitic scattering. The thickness of the wall $(0.01 \mathrm{~mm})$ that is characterized by poor lateral (along the capillary) thermal conductivity, also guarantees minimum thermal losses (Keller et al., 1998), optimizing the thermal recordings. The AMF samples were heated in the calorimeter at $60^{\circ} \mathrm{C}$ for $10 \mathrm{~min}$ to melt all existing nuclei. The AMF samples were cooled at 3 and $1{ }^{\circ} \mathrm{C} / \mathrm{min}$ from 60 to $-10^{\circ} \mathrm{C}$. X-ray diffraction patterns were recorded with a time frame of $60 \mathrm{~s}$, and DSC signal was recorded every 3 s during cooling, using a single computer for thermal control and data acquisition. Following cooling, the samples of AMF were heated from -10 to $60^{\circ} \mathrm{C}$ at $2^{\circ} \mathrm{C} / \mathrm{min}$ with an XRD time frame of $60 \mathrm{~s}$ and DSC signal recorded every $3 \mathrm{~s}$.

Each XRD pattern was analyzed using PEAKFIT software (JANDEL Scientific, Erkrath, Germany). The XRD peaks were fitted by Gaussian-Lorentzian (sum) equation as described in Lopez et al. (2001d) to determine the position of the maximum and the maximal intensity of each peak as a function of $\mathrm{T}$.

\section{DSC Measurements}

Thermal analyses were conducted with a DSC-7 Perkin Elmer (St. Quentin en Yvelines, France) using aluminum pans of $50 \mu \mathrm{L}$ [pan (part \#B014-3021) and cover (part \#B014-3004)] hermetically sealed. The reference was an empty, hermetically sealed aluminum pan. The AMF samples were heated at $60^{\circ} \mathrm{C}$ during 5 min to melt all crystals and nuclei. Crystallization curves were recorded from 60 to $-8^{\circ} \mathrm{C}$ at different cooling rates: $0.5,1$, 2,3 , and $5^{\circ} \mathrm{C} / \mathrm{min}$. Then, following cooling, all melting curves were recorded from -8 to $60^{\circ} \mathrm{C}$ at $2^{\circ} \mathrm{C} / \mathrm{min}$. The calibration of the calorimeter was controlled with lauric acid melting (melting point $=43.7^{\circ} \mathrm{C}$; melting enthalpy = $8.53 \mathrm{Kcal} / \mathrm{mol})$. It is worth noting that the aluminum pans used in this study likely induce different nucleation sites than glass capillaries as crystallization is observed at a lower T. This is likely related to the nature of the chemical species found at the surface of both materials.

\section{Polarized Light Microscopy}

Anhydrous milk fat was examined by microscopy between crossed polarizers and with a $\lambda / 4$ retarder in white light using a NIKON E600 Eclipse direct microscope (Champigny/Marne, France) equipped with a long focus objective $40 \times 0.55$; 0 to $2 \mathrm{~mm}$ ). NIKON Coolpik 950 camera is used as a picture recorder with a resolution of $1600 \times 1200$ pixels. A homemade sample holder was used to monitor the sample $\mathrm{T}$ between -10 and $60^{\circ} \mathrm{C}$. Briefly, the sample is placed between 2 circular lamellae located in a cavity of a Peltier-cooled stage, the $\mathrm{T}$ of which is controlled at $\pm 0.2^{\circ} \mathrm{C}$. All samples were heated to $+60^{\circ} \mathrm{C}$ before being cooled at $0.2,1$, and $3^{\circ} \mathrm{C} / \mathrm{min}$ or quenched to $-8^{\circ} \mathrm{C}$ on the microscope stage.

\section{RESULTS AND DISCUSSION}

\section{Crystallization Behavior of AMF at Intermediate Cooling Rates}

Crystallization of AMF was studied at intermediate cooling rates by coupled XRDT and high-sensitivity DSC techniques, simultaneously on the same sample.

Cooling at $3^{\circ} \mathbf{C} / \mathbf{m i n}$. The XRD patterns recorded simultaneously at small and wide angles as a function of T during cooling of AMF from 60 to $-10^{\circ} \mathrm{C}$ (Figure 1) could be divided in $2 \mathrm{~T}$-delimited domains. In the $60 \geq$ $\mathrm{T} \geq 19^{\circ} \mathrm{C}$ domain, XRD patterns recorded at both small (Figure 1) and wide (Figure 1, insert) angles show that milk fat TG are in their liquid state. Indeed, at small angles, no diffraction lines are recorded, and at wide angles, the x-ray bump recorded at about $q=1.396 \AA^{-1}$ $(4.5 \AA)$ is related to x-ray scattering from the liquidcrystalline organization of TG (Larsson, 1972). For T $\leq$ $16^{\circ} \mathrm{C}$, the recordings of diffraction lines at both small and wide angles correspond to TG crystallization. At wide angles (Figure 1, insert), the single peak of diffraction recorded at about $q=1.514 \AA^{-1}(4.15 \AA)$ is characteristic of TG crystallization with a hexagonal packing of the acylglycerol chains. At small angles (Figure 1), TG crystallization is related to the recording of XRD peaks at about $q=0.136 \AA^{-1}(46 \AA)$ and $0.163 \AA^{-1}(38.5 \AA)$ with an increase of $x$-ray scattering at very small angles $(q=$ $\left.0.05 \AA^{-1}\right)$. It is worth noting that the line at $q=0.136$ $\AA^{-1}$ is observed slightly before that of $q=0.163 \AA^{-1}$ (see subsequent DSC recording interpretation). From about $10^{\circ} \mathrm{C}$, sharp diffraction lines are simultaneously recorded at $\mathrm{q}=0.0873 \AA^{-1}(72 \AA), 0.1745 \AA^{-1}(36 \AA), 0.2618$ $\AA^{-1}(24 \AA)$, and $0.4363 \AA^{-1}(14.4 \AA)$. All of the diffraction lines increase in intensity as a function of the decrease in $\mathrm{T}$.

All XRD patterns recorded at small and wide angles during cooling of $\mathrm{AMF}$ at $3^{\circ} \mathrm{C} / \mathrm{min}$ were analyzed to determine the position and the maximal intensity of each diffraction peak as a function of $\mathrm{T}$ to delimit the domains of existence of the crystalline varieties. Both intensity evolution plots are presented in Figure 2 (A and B, respectively). 


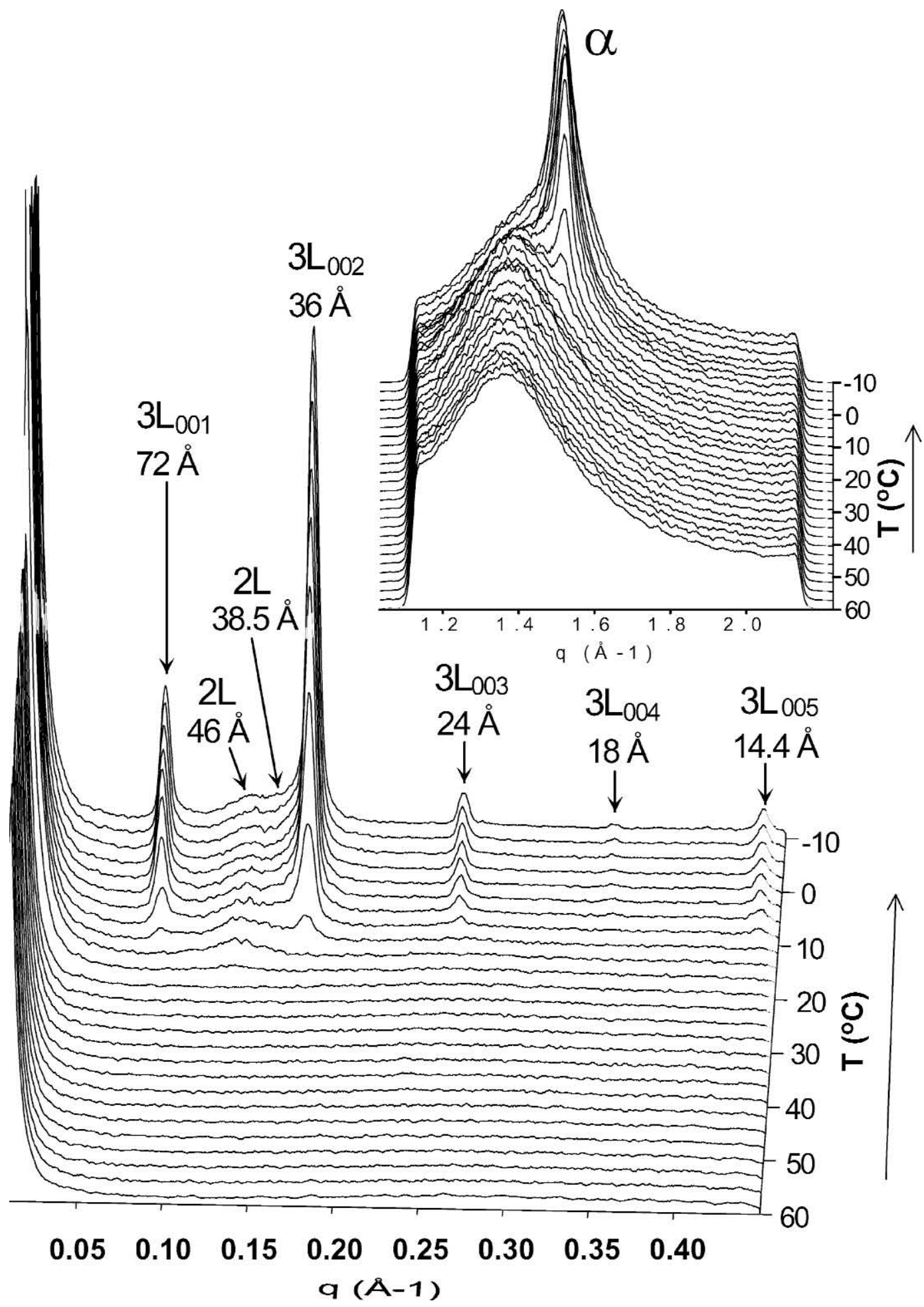

Figure 1. Three-dimensional plots of small and wide (insert) angle x-ray diffraction patterns recorded simultaneously during cooling of anhydrous milk fat at $-3^{\circ} \mathrm{C} / \mathrm{min}$ from 60 to $-10^{\circ} \mathrm{C}$. The types of longitudinal double (2L) and triple (3L) chain lengths, as well as their associated long spacings, and lateral packing (insert) are indicated on the figure. $q=$ Scattering vector; $\mathrm{T}=$ temperature . 


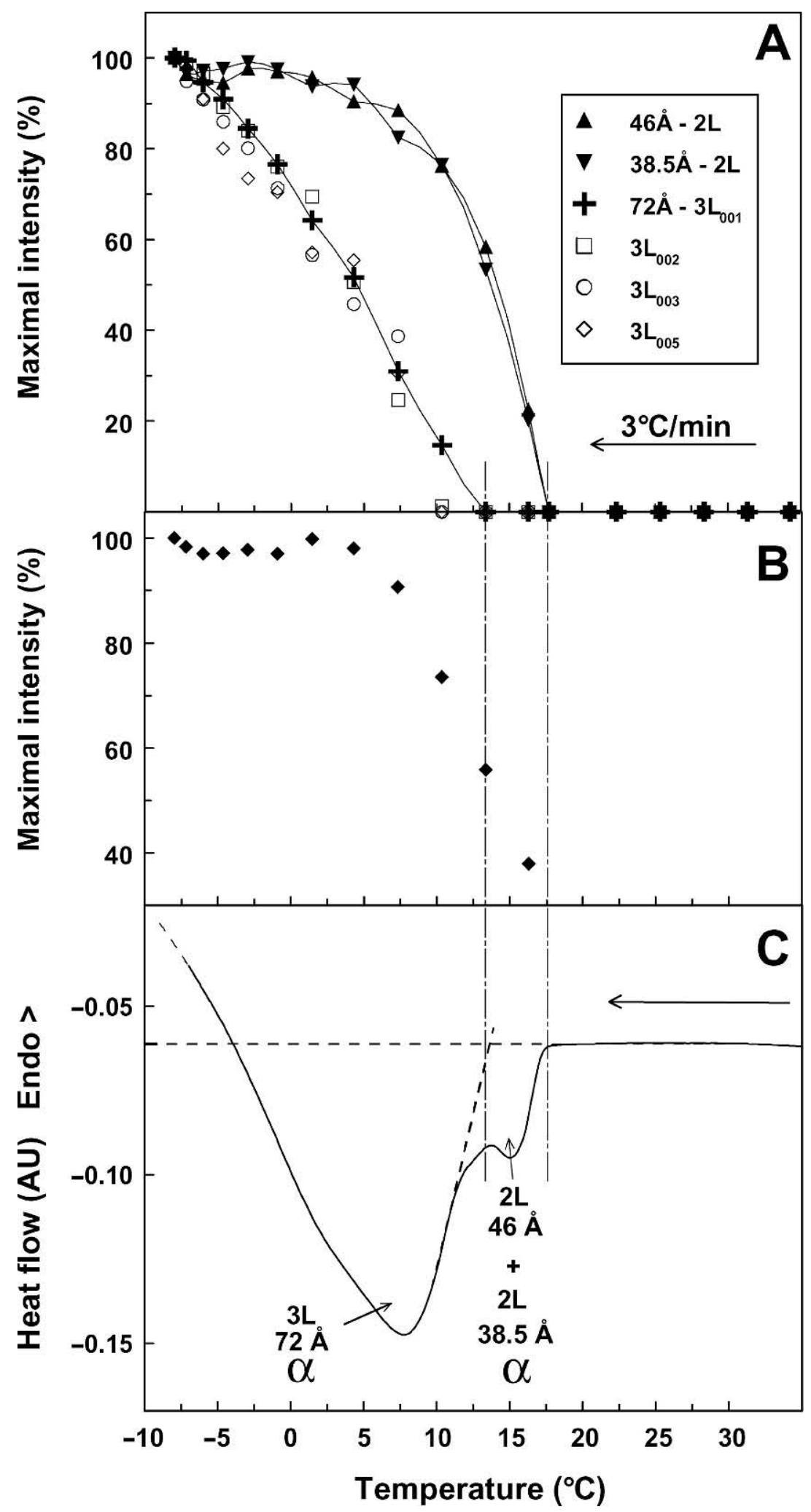

Figure 2. Analysis of x-ray diffraction (XRD) peaks (shown Figure 1) as a function of temperature and differential scanning calorimetry (DSC) recording. Intensity evolutions of the long and short spacings of milk fat as determined by A) small- and B) wide-angle XRD and C) DSC recordings observed during cooling of anhydrous milk fat at $-3^{\circ} \mathrm{C} / \mathrm{min}$ from 40 to $-10^{\circ} \mathrm{C}$. All recordings were simultaneously obtained from the same sample. $\mathrm{AU}=$ arbitrary unit; $2 \mathrm{~L}=$ bi-layered stacking, and $3 \mathrm{~L}=$ tri-layered stacking. 


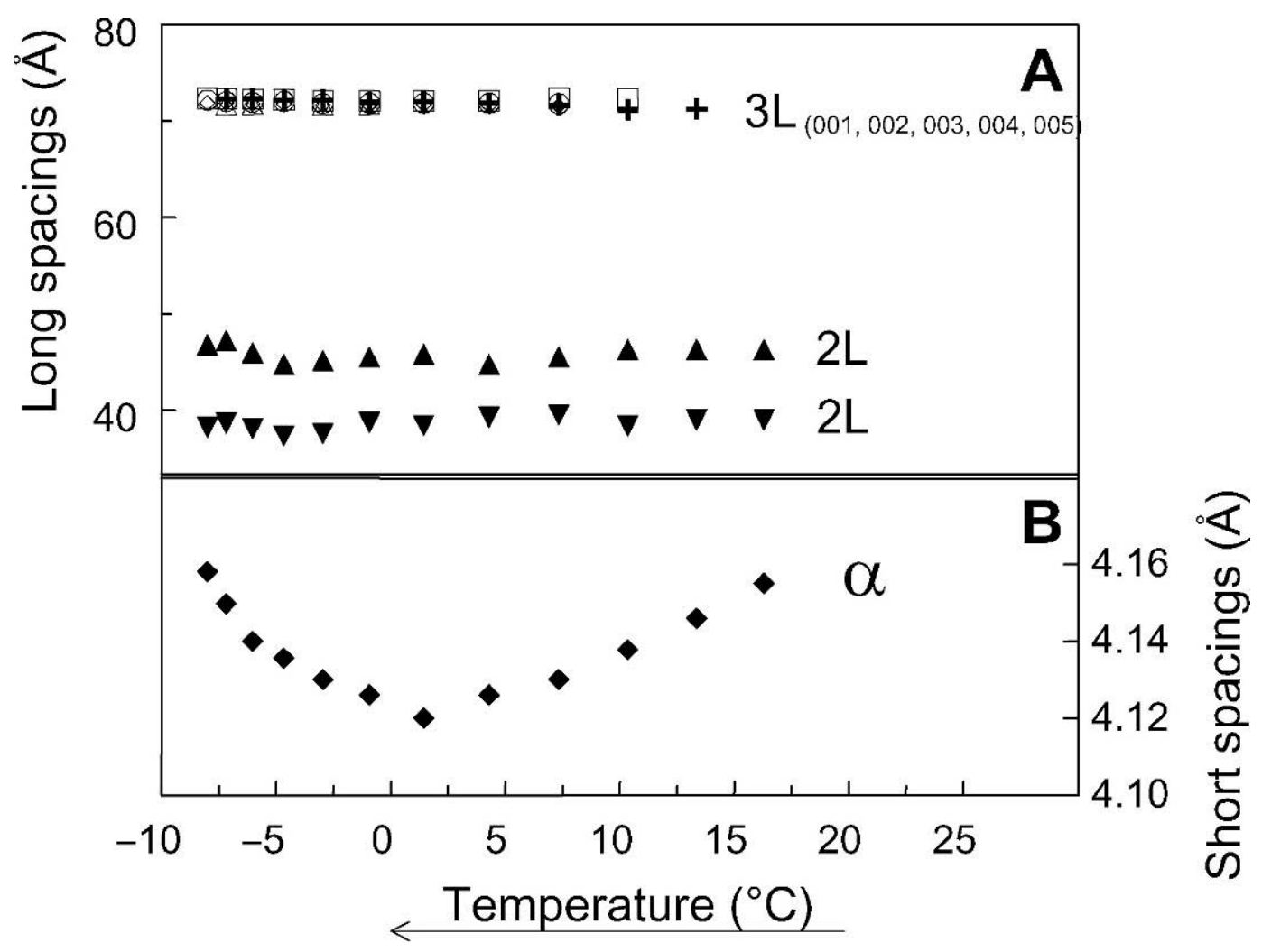

Figure 3. Evolution of the long (A) and short (B) spacings as determined by small- and wide-angle x-ray diffraction, respectively, during cooling of anhydrous milk fat at $-3^{\circ} \mathrm{C} / \mathrm{min}$ (for higher orders, the repeat period has been multiplied by the corresponding reflection order). $2 \mathrm{~L}=$ bi-layered stacking; $3 \mathrm{~L}=$ tri-layered stacking.

On cooling at $3^{\circ} \mathrm{C} / \mathrm{min}$, crystallization of $\mathrm{AMF}$ occurs from about $17^{\circ} \mathrm{C}$ with the successive formation of 2 lamellar structures with double-chain length organizations $(\mathbf{2 L})$ of about 46 , then $38.5 \AA$, both with a hexagonal packing of the acylglycerol chains ( $\alpha$ form). Then, from about $14^{\circ} \mathrm{C}$, the simultaneous increases in intensity of a new series of diffraction lines recorded at small angles mean that they correspond to the formation of the same crystalline organization with different orders of diffraction, namely a lamellar structure with a triple-chain length organization $(\mathbf{3 L})$ of $72 \AA$ and a hexagonal packing of $\alpha$ type. Crystallization of TG in the lowest degree of organization, namely the unstable $\alpha$ form, is not surprising in the conditions of cooling used.

The evolutions, as a function of $\mathrm{T}$, of the long and short spacings recorded at small and wide angles, respectively, during cooling of $\mathrm{AMF}$ at $3^{\circ} \mathrm{C} / \mathrm{min}$ are presented Figure 3.

The long spacings of the diffraction lines related to the $3 \mathrm{~L}(72 \AA)$ variety are multiplied by their corresponding order of diffraction. The superimposition of these long spacings confirms that they belong to the same crystalline structure. Moreover, this figure shows that the long spacings do not show any significant evolution as a function of T. On the contrary, the short spacing corresponding to $\alpha$ packing first decreases from $4.15 \AA$ $\left(16^{\circ} \mathrm{C}\right)$ to $4.13 \AA\left(-1^{\circ} \mathrm{C}\right)$, meaning that the hexagonal organization of the acylglycerol chains becomes more dense than less dense upon increasing up to $4.16 \AA$ $\left(-8^{\circ} \mathrm{C}\right)$. The period of evolution observed is similar to that observed in cream (Lopez et al., 2001a) for which we concluded to the formation of sub- $\alpha$ form at the end of cooling in the same conditions. Although the absence of a second broad peak at about $3.8 \AA$ prevented us from reaching the same conclusion, its observation at the beginning of heating indicated that its formation was time dependent, as has already observed in cream (Lopez et al., 2001a) (Figure 4).

The crystallization curve recorded by DSC simultaneously with XRDT experiments during the cooling of AMF at $3^{\circ} \mathrm{C} / \mathrm{min}$ is presented in Figure 2C. Coupling of XRDT and DSC measurements allows us to relate the structural and the thermal behavior and then to identify the DSC peaks. From the initial T of crystallization measured at $17.2^{\circ} \mathrm{C}, 3$ overlapped, exothermal events are recorded on the crystallization curve. The first exotherm is related to crystallization of the $\alpha 2 \mathrm{~L}$ (46 $\AA$ ) variety; that of the $\alpha 2 \mathrm{~L}(38.5 \AA$ ) is slightly delayed (Figure 1; 


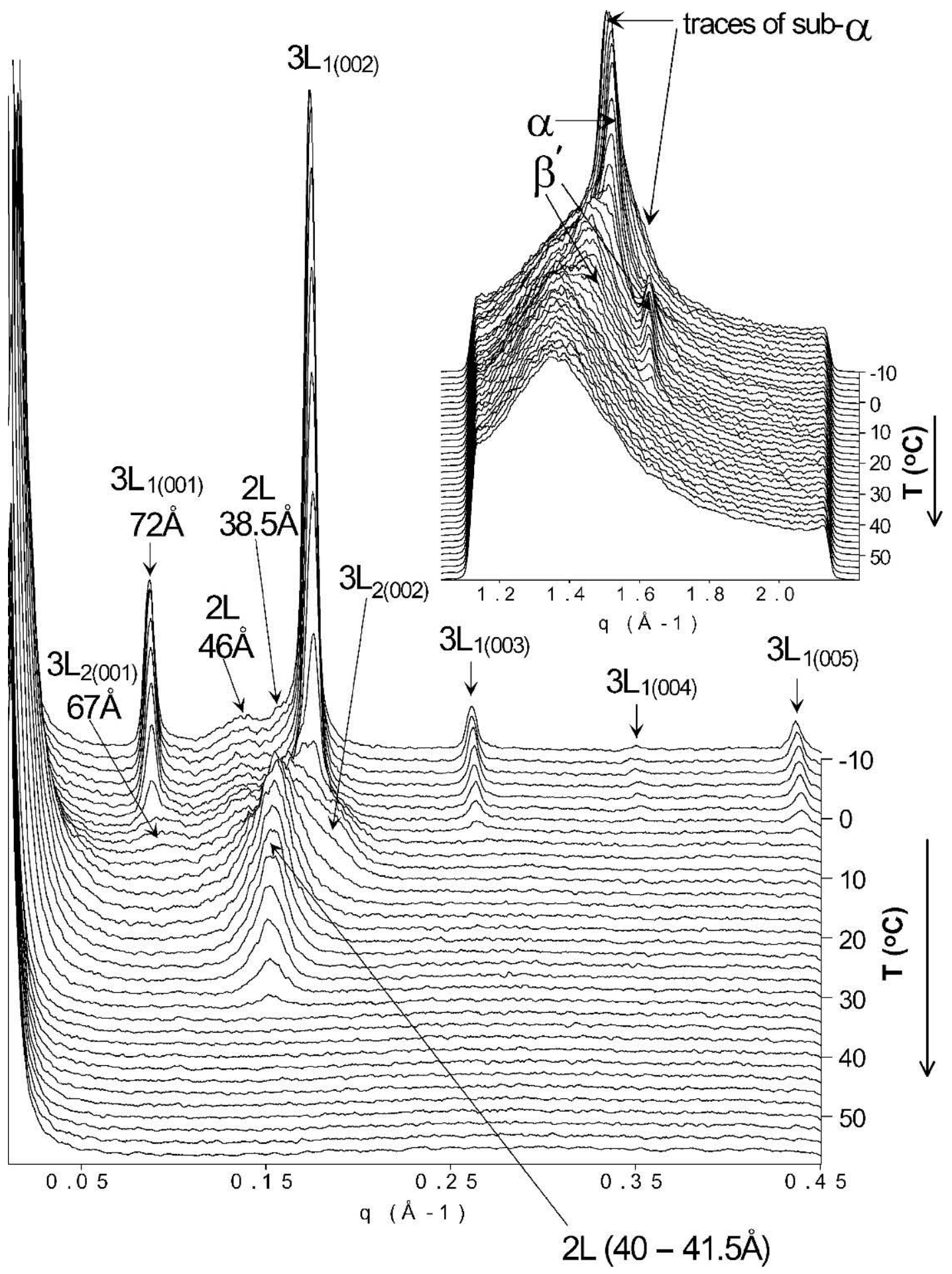

Figure 4. Three-dimensional plots of small- and wide- (insert) angle x-ray diffraction patterns recorded simultaneously during heating of anhydrous milk fat at $2^{\circ} \mathrm{C} / \mathrm{min}$ from -10 to $60^{\circ} \mathrm{C}$, following a cooling at $3^{\circ} \mathrm{C} / \mathrm{min}$ (Figures 1 to 3 ). The types of longitudinal double (2L) and triple (3L) chain lengths, as well as their associated long spacings, and lateral packing (insert) are indicated on the figure. $\mathrm{q}=\mathrm{Scattering}$ vector; $\mathrm{T}=$ temperature. 
Figure 2C). The third exotherm corresponds to crystallization of the $\alpha 3 \mathrm{~L}(72 \AA)$ structure with an initial T of crystallization measured at $13.7^{\circ} \mathrm{C}$.

Heating at $2^{\circ} \mathbf{C} / \mathbf{m i n}$. After cooling at $3^{\circ} \mathrm{C} / \mathrm{min}$, the AMF sample was immediately (after $\sim 30 \mathrm{~s}$ ) heated from -10 to $60^{\circ} \mathrm{C}$ at $2^{\circ} \mathrm{C} / \mathrm{min}$ to study the polymorphic evolution of the crystalline structures formed.

The XRDT patterns recorded simultaneously at small and wide angles during heating of AMF are plotted as 3 -D views (Figure 4 ). Small-angle XRD patterns could be divided into 4 T-delimited domains. In the first domain, the diffraction peaks formed on cooling decrease in intensity. Then, in the $6<\mathrm{T}<14^{\circ} \mathrm{C}$ domain, complex phase transitions are observed. A single line of diffraction corresponds to the formation, and the successive melting of a double-chain length structure $(2 \mathrm{~L})$ is recorded until final melting of all TG at $\mathrm{T}>34^{\circ} \mathrm{C}$. At wide angles, the following transitions are observed, starting from traces of sub- $\alpha$ : sub- $\alpha \rightarrow \alpha \rightarrow \beta^{\prime} \rightarrow$ liquid.

The XRD patterns recorded during heating of AMF at $2^{\circ} \mathrm{C} / \mathrm{min}$ were analyzed using PEAKFIT software to determine the position of the maximum of each peak recorded as well as its maximal intensity. The evolutions of the maximal intensities of the XRD peaks recorded both at small and wide angles are plotted in the same graph as a function of $T$ to relate the longitudinal organization to the lateral packing of the TG molecules in lamellar structures (Figure 5).

The evolutions of the small-angle peaks show successive meltings and phase transitions of the crystalline structures. First, the decreases in intensity of the diffraction peaks associated with the $\alpha 3 \mathrm{~L}(72 \AA)$ correspond to the melting of this crystalline structure. In the $6<\mathrm{T}$ $<12^{\circ} \mathrm{C}$ domain, the simultaneous formation of broad diffraction peaks at $q=0.094 \AA^{-1}(67 \AA)$ and $0.188 \AA^{-1}$ $(33.4 \AA)$ corresponds to the formation of a $3 \mathrm{~L}(67 \AA)$ structure with its second order of diffraction $\left(3 \mathrm{~L}_{2(002)}\right)$. This structure, which melts at $\mathrm{T}>12^{\circ} \mathrm{C}$, may originate from a $3 \mathrm{~L}(72 \AA) \rightarrow 3 \mathrm{~L}(67 \AA)$ transition during heating. The 2L lamellar structures first formed on cooling with thicknesses of 46 and $38.5 \AA$ melt at $\mathrm{T}>16^{\circ} \mathrm{C}$.

At $\mathrm{T}>8^{\circ} \mathrm{C}$, SAXD patterns show the formation of a new double-chain length organization. This long spacing may be composed of TG initially incorporated in the $3 \mathrm{~L}$ (72 $\AA$ ) structure. Likely, the melting of the $3 \mathrm{~L}(72 \AA)$ variety leads to $\mathrm{TG}$ in the liquid state and to a rearrangement of TG to form both the $3 \mathrm{~L}(67 \AA)$ and the new $2 \mathrm{~L}$ organization. At about $12^{\circ} \mathrm{C}$, the inflexion point recorded in the evolution in intensity of the $2 \mathrm{~L}$ variety is correlated with the melting of the $3 \mathrm{~L}(67 \AA)$, the $2 \mathrm{~L}$ (46 $\AA$ ), and the $2 \mathrm{~L}(38.5 \AA$ ) structures. Likely, some TG originating from these 3 organizations incorporate into the new 2L stacking. Such rearrangements of the TG molecules into a lamellar organization are facilitated by

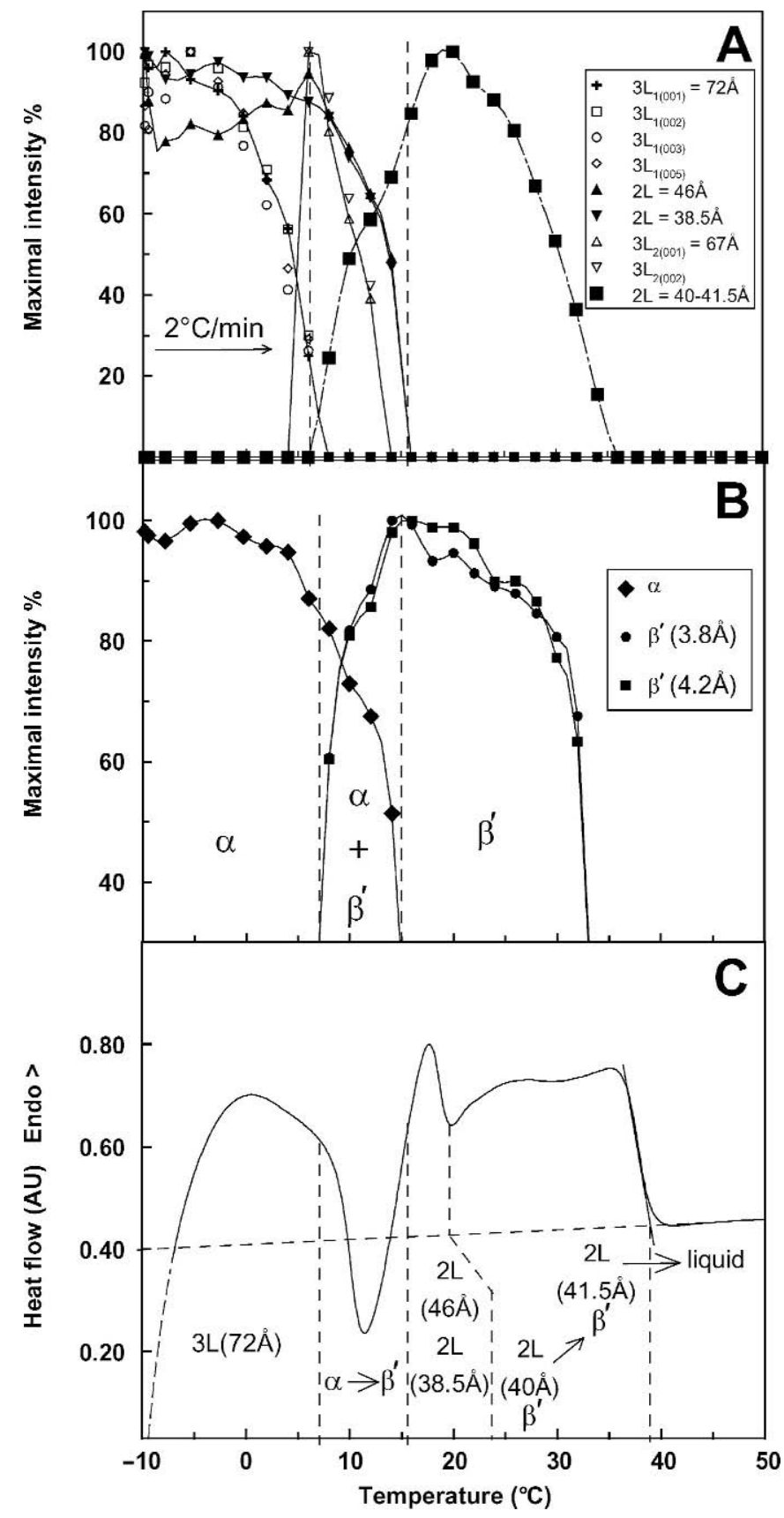

Figure 5. Evolution of intensities of $x$-ray diffraction (XRD) peaks (shown Figure 4) as a function of temperature and analysis of the differential scanning calorimetry (DSC) recorded simultaneously to XRDT experiments on the same sample. Evolution of the long and short spacings of milk fat as determined by (A) small- and (B) wideangle XRD recordings during heating of anhydrous milk fat at $2^{\circ} \mathrm{C} /$ min from -10 to $50^{\circ} \mathrm{C}$ and DSC recordings $(\mathrm{C})$. $\mathrm{AU}=$ arbitrary unit; $2 \mathrm{~L}=$ bi-layered stacking, and $3 \mathrm{~L}=$ tri-layered stacking.

the presence of a liquid phase. The $2 \mathrm{~L}$ variety takes advantage of the melting of the other crystalline structures for its formation until $20^{\circ} \mathrm{C}$. Then, the decrease in intensity of the diffraction line associated to this $2 \mathrm{~L}$ 
longitudinal stacking corresponds to its progressive melting until its disappearance at $\mathrm{T}>34^{\circ} \mathrm{C}$. At wide angles, the meltings of the $3 \mathrm{~L}(72 \AA)$ and $2 \mathrm{~L}(46 \AA+$ $38.5 \AA$ ) varieties are correlated with the decrease in intensity of the $\alpha$ form. The formation of the new $2 \mathrm{~L}$ structure is correlated with the recording of 2 diffraction peaks at about 3.8 and $4.2 \AA$, characteristic of the formation of a $\beta^{\prime}$ form. These results show that a $\alpha \rightarrow \beta^{\prime}$ polymorphic transition occurs during heating of $\mathrm{AMF}$ at $2^{\circ} \mathrm{C} / \mathrm{min}$.

As a summary, the analysis of the XRD data corresponding to the longitudinal (Figure 5A) and the lateral (Figure 5B) organizations of TG molecules within crystals recorded during heating of AMF leads to the following polymorphic transitions:

$$
\begin{aligned}
& \alpha 3 \mathrm{~L}(72 \AA)+\alpha 2 \mathrm{~L}(46 \AA)+\alpha 2 \mathrm{~L}(38.5 \AA) \rightarrow \\
& \beta^{*} 3 \mathrm{~L}(67 \AA)+\beta^{\prime} 2 \mathrm{~L}(40 \text { to } 41.5 \AA) \rightarrow \text { liquid. }
\end{aligned}
$$

The DSC curve recorded simultaneously with XRDT experiments is presented Figure 5C. The plot of XRDT data obtained from analysis of the patterns recorded at both small and wide angles and DSC data on the same $\mathrm{T}$ scale allows us to identify the thermal events. On the melting curve recorded, 3 endothermic peaks corresponding to the low melting point, medium melting point, and high melting point fractions are recorded together with an exotherm between the endotherms of the low and medium melting point fractions. The correlations between XRD and DSC data show that the first endotherm recorded on heating (low melting point fraction) corresponds to the melting of the $3 \mathrm{~L}(72 \AA)$ structure. The exotherm recorded between about 10 and $14^{\circ} \mathrm{C}$ by coupled DSC is related to the $\alpha \rightarrow \beta^{\prime}$ phase transition. The endotherm recorded between $\sim 14$ and $20^{\circ} \mathrm{C}$ (medium melting point fraction) is related to the melting of both the $2 \mathrm{~L}(46 \AA)$ and the $2 \mathrm{~L}(38.5 \AA)$ structures. Finally, the endothermic, overlaped peaks recorded (high melting point fraction) corresponds to the progressive melting of the $2 \mathrm{~L}$ structure until final melting of all AMF TG at $\mathrm{T}>39^{\circ} \mathrm{C}$.

The numerous transitions observed both by DSC, including an exothermic peak, and by XRDT, especially at small angles, demonstrate that several crystalline reorganizations occur during heating at $2^{\circ} \mathrm{C} / \mathrm{min}$ following a cooling at $-3^{\circ} \mathrm{C} / \mathrm{min}$. This is a consequence of the formation of very unstable varieties at this fast cooling rate.

Cooling at $1^{\circ} \mathrm{C} / \mathbf{m i n}$. The structural behavior of AMF was monitored on cooling at $-1^{\circ} \mathrm{C} / \mathrm{min}$. Then, the evolu-

*The exact subcell packing of the phase displaying a 3L stacking at $67 \AA$ is not clearly established (Figure 5). tion of the crystalline structures formed was studied on a following heating at $2^{\circ} \mathrm{C} / \mathrm{min}$. The XRD patterns recorded at both small and wide angles as a function of T during the cooling and the heating of the same sample of AMF are presented in Figure 6.

Compared with a cooling at $-3^{\circ} \mathrm{C} / \mathrm{min}$, similar crystalline structures are formed. However, decreasing the cooling rate from -3 to $-1^{\circ} \mathrm{C} / \mathrm{min}$ favors the formation of the more stable $2 \mathrm{~L}(46 \AA)$ variety. The important variations of the slope of the central scattering at edges of the beam stop trace (at $q<0.05 \AA^{-1}$ ), observed on both cooling and heating recordings (Figure $6 \mathrm{~B}$ ), were related to the crystal size and number formed. The variations of the central scattering are also strongly influenced by the rate of cooling; the larger the crystals, the steeper the slope, in agreement with microscopy observations.

\section{Influence of Cooling Rate}

Thermal behavior. The thermal behavior of AMF was studied by simple DSC at different cooling rates, with $5 \geq|\mathrm{dT} / \mathrm{dt}| \geq 0.5^{\circ} \mathrm{C} / \mathrm{min}$ (Figure $7 \mathrm{~A}$ ) (Ollivon 1982; Lavigne, 1995). Following crystallization, all of the samples of AMF were heated at the same rate, $|\mathrm{dT} / \mathrm{dt}|=2^{\circ} \mathrm{C} /$ min, to study the influence of the cooling rate on the melting behavior of AMF TG (Figure 7B). Both sets of recordings show that the initial $T$ of crystallization measured and the shape of both the crystallization and melting curves depend on the cooling rate. The initial $\mathrm{T}$ of crystallization of milk fat decreases with increasing cooling rate (Table 1), in relation to the nucleation process. On cooling, the authors generally consider the existence of 2 exothermic process that correspond to crystallization of 2 groups of TG (Lavigne, 1995; ten Grotenhuis et al., 1999). However, our results show the existence of 3 exotherms and even 4 . The exotherm formed between the 2 others is hardly detected at $|\mathrm{dT} / \mathrm{dt}|=5^{\circ} \mathrm{C} / \mathrm{min}$. Thus, its characterization needs a good resolution and available experimental conditions.

The DSC melting curves recorded during heating of $\mathrm{AMF}$ at $2{ }^{\circ} \mathrm{C} / \mathrm{min}$ after the samples were cooled at different cooling rates varying from 0.5 to $5^{\circ} \mathrm{C} / \mathrm{min}$ are shown in Figure 7B. The curves corresponding to AMF samples cooled at $-1,-2,-3$, and $-5^{\circ} \mathrm{C} / \mathrm{min}$ are similar, showing the 3 distinctive endothermic peaks corresponding to the low, medium, and high melting point fractions. Similar results were obtained by ten Grotenhuis et al. (1999) with DSC melting curves recorded at $5^{\circ} \mathrm{C} / \mathrm{min}$ after cooling of milk fat at $-1,-2.5,-5$, and $-10^{\circ} \mathrm{C} / \mathrm{min}$. The exotherms, which delimit endothermal melting transitions, are more clearly evidenced for faster cooling rates (Figure 7B). This is interpreted as the faster the cooling rate the more metastable state formed, and the more intense the relaxation phenomenon. The final melting $\mathrm{T}$ ob- 

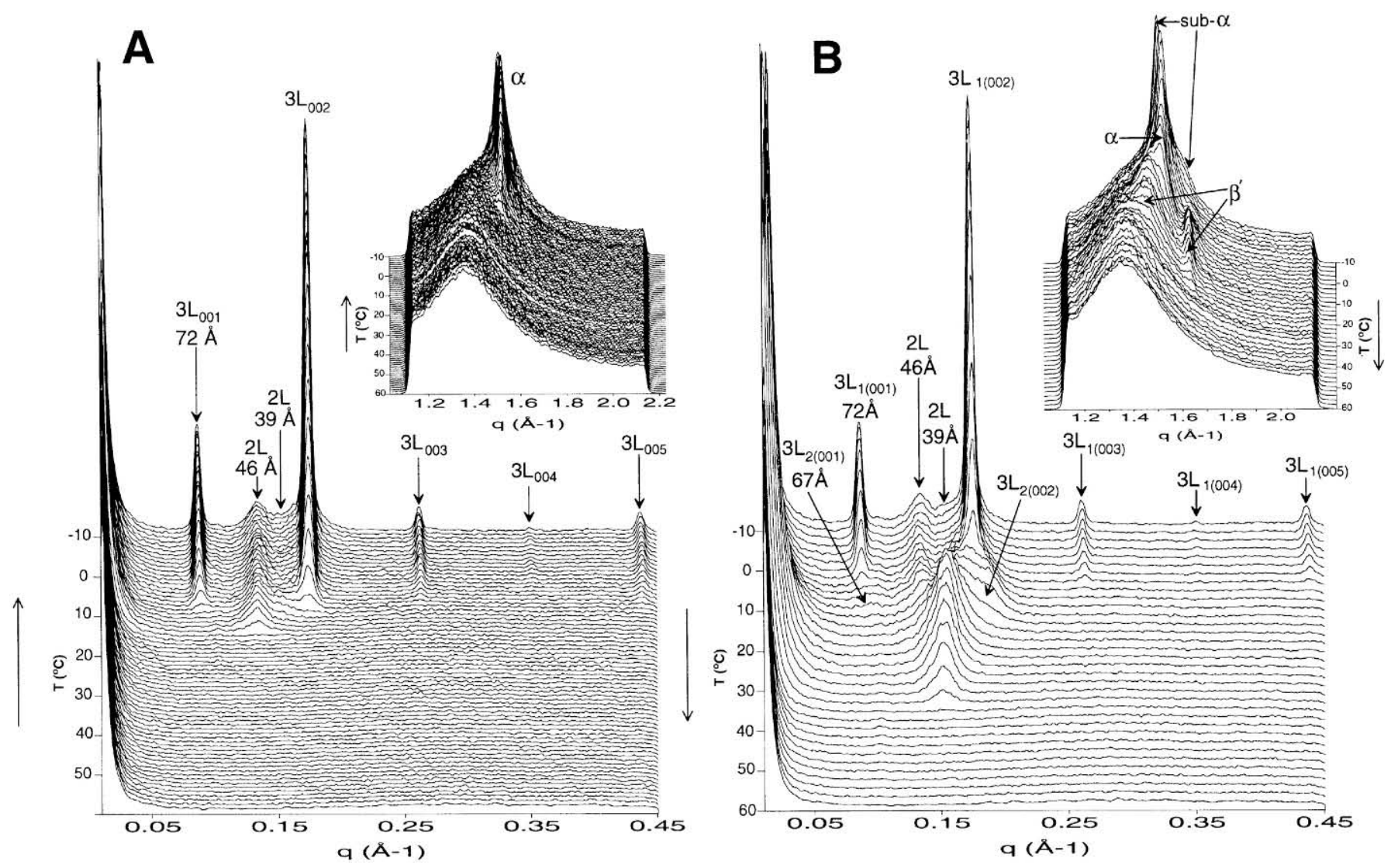

Figure 6. Three-dimensional plots of small- and wide- (insert) angle X-ray diffraction patterns recorded simultaneously during the cooling at $-1^{\circ} \mathrm{C} / \mathrm{min}$ from 60 to $-10^{\circ} \mathrm{C}$ (A) and the following heating at $2^{\circ} \mathrm{C} / \mathrm{min}$ from -10 to $60^{\circ} \mathrm{C}$ (B) of anhydrous milk fat. The types of longitudinal double (2L) and triple (3L) chain lengths, as well as their associated long spacings, and lateral packing (inserts) are indicated on the figures. $q=$ Scattering vector; $\mathrm{T}=$ temperature.

served as a function of cooling rate (Table 1) are not significantly different; that observed on cooling clearly reflects an influence of nucleation process.

The crystallizations of only 2 groups of TG on cooling, but the melting of 3 on heating, necessarily imply the formation of several groups, at least 3 , of mixed crystals. The broad exotherm recorded during cooling shows that the larger group of these mixed crystals is probably constituted by mixed types (with respect to unsaturation and chain length) of TG molecules. Indeed, the melting of this mixed crystal is progressive and leads to broad endotherms then to the formation of a liquid phase in addition to the crystals. Also, the exothermal processes observed on heating are related to the formation of liquid phases. This liquid phase favors structural rearrangements in relation to the monotropic behavior of TG, as mixed crystals may dissolve in and transform into a new polymorphic form.

The formation of mixed polymorphic crystals in milk fat has already been reported (Mulder, 1953; van Beresteyn, 1972; Mulder and Walstra, 1974). On fast cooling, likely a large number of low-melting TG are trapped into a non-selective crystal lattice initially formed by highmelting TG, and slow cooling promotes fractionation of the fat.

Structural behavior. Figure 8 illustrates the various thermal treatments that were applied to AMF samples in the different studies of the series to analyze, by XRDT and DSC techniques, the crystalline structures formed, as well as their phase transitions (Table 2).

Figure 9 (A and B, respectively) represents the SAXD and WAXD patterns recorded at $-8^{\circ} \mathrm{C}$ after cooling at the rates indicated Figure 8. Their comparisons show that the thermal treatment applied directly influences the crystalline structures formed by TG in bulk.

Cooling of AMF in extremely fast conditions, by quenching (at about $-1500^{\circ} \mathrm{C} / \mathrm{min}$ ), or at intermediate cooling rates, -1 and $-3^{\circ} \mathrm{C} / \mathrm{min}$, first leads to the crystallization of $\alpha$ form with the coexistence of 2 longitudinal stackings of the TG molecules corresponding to tripleand double-chain length structures (Figure 9A). In isothermal conditions at about $-10^{\circ} \mathrm{C}$, this initial variety 

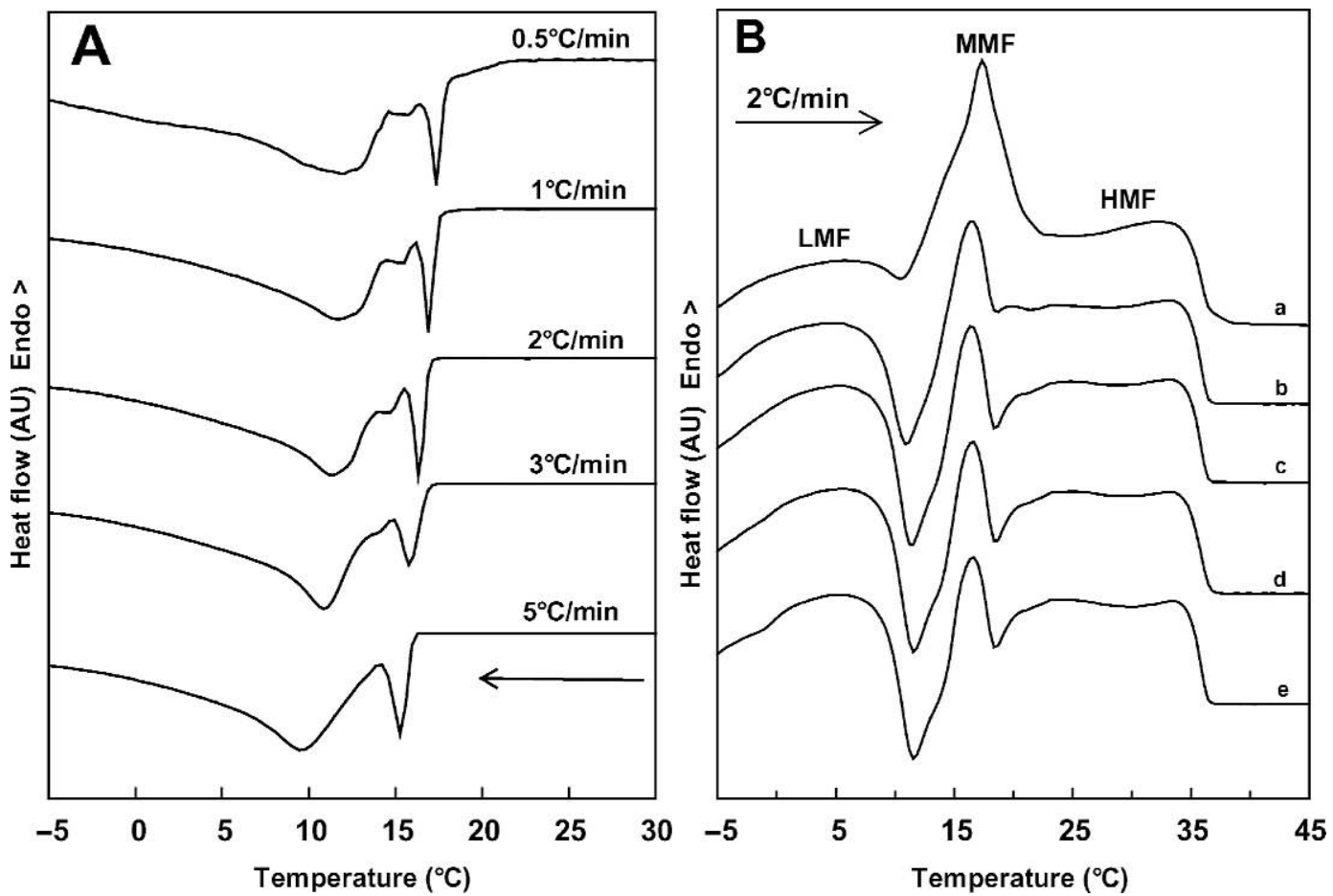

Figure 7. Differential scanning calorimetry recordings observed as a function of cooling rate for anhydrous milk fat (AMF). A) Crystallization curves recorded during cooling of $\mathrm{AMF}$ from 60 to $-10^{\circ} \mathrm{C}$ at the different rates indicated on the figure. B) Melting curves recorded during the following heating of the samples at $2^{\circ} \mathrm{C} / \mathrm{min}$ after the cooling at $0.5^{\circ} \mathrm{C} / \mathrm{min}(\mathrm{a}), 1^{\circ} \mathrm{C} / \mathrm{min}(\mathrm{b}), 2^{\circ} \mathrm{C} / \mathrm{min}(\mathrm{c}), 3^{\circ} \mathrm{C} / \mathrm{min}(\mathrm{d})$, and $5^{\circ} \mathrm{C} /$ $\min (\mathrm{e})$.

progressively transforms into sub- $\alpha$ form, following a time-dependent process, as it is observed at the beginning of the heating and it was not at the end of the cooling (Figures 1 and 4). The small bump observed at about 3.8 A on Figure 9B for quenched sample and the shift of the peak position shows that the process of the $\alpha \rightarrow$ sub- $\alpha$ transition likely takes about $3 \mathrm{~min}$ at $-8^{\circ} \mathrm{C}$. The observation of such a transition was not clearly reported before (Lopez et al., 2001b).

Quenching of $\mathrm{AMF}$ at about $1000^{\circ} \mathrm{C} / \mathrm{min}$ (Lopez et al., $2001 b)$ allowed us to identify a metastable $2 \mathrm{~L}(47 \AA)$ structure characterized by the formation of a sharp dif-

Table 1. Variation of the crystallization onset and final melting points observed after cooling at different rates and melting at $2^{\circ} \mathrm{C} / \mathrm{min}$, respectively.

\begin{tabular}{lll}
\hline $\begin{array}{l}\text { Cooling rate } \\
{[\mathrm{dT} / \mathrm{dt}} \\
\left.\left({ }^{\circ} \mathrm{C} / \mathrm{min}\right)\right]\end{array}$ & $\begin{array}{l}\text { Initial } \\
\text { temperature of } \\
\text { crystallization } \\
\left({ }^{\circ} \mathrm{C}\right)\end{array}$ & $\begin{array}{l}\text { Final } \\
\text { melting } \\
\text { temperature } \\
\left({ }^{\circ} \mathrm{C}\right)\end{array}$ \\
\hline 5 & 16.05 & 37.48 \\
4 & 16.46 & 37.56 \\
3 & 16.92 & 37.61 \\
2 & 17.03 & 37.49 \\
1 & 17.60 & 37.58 \\
0.5 & 18.02 & 37.77 \\
\hline
\end{tabular}

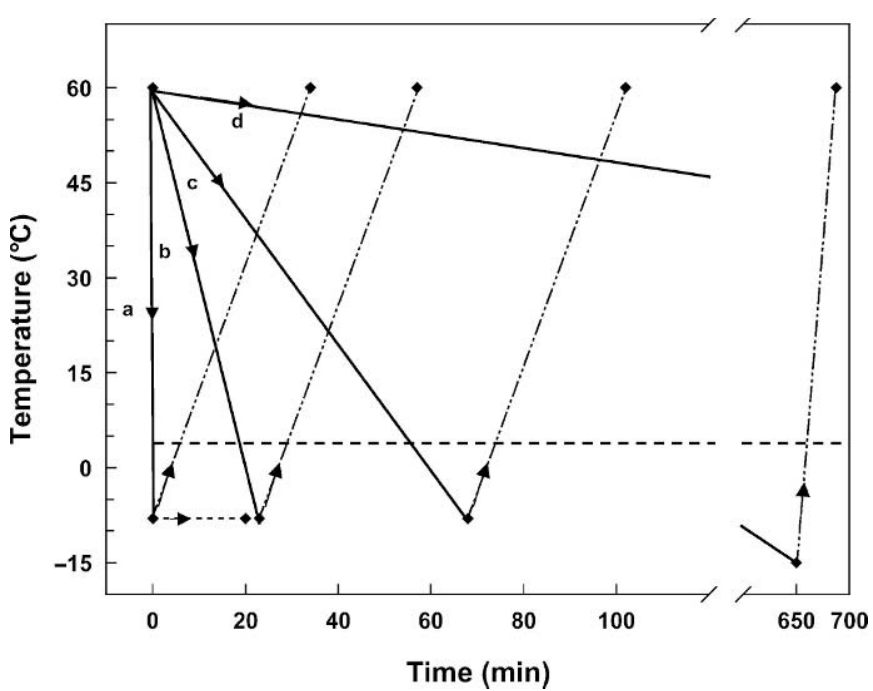

Figure 8. Schematic representation of the thermal treatments applied at different rates on cooling [a) quenching, b) $-3^{\circ} \mathrm{C} / \mathrm{min}, \mathrm{c}$ ) $-1^{\circ} \mathrm{C} / \mathrm{min}$, and d) $-0.1^{\circ} \mathrm{C} / \mathrm{min}$ ] to anhydrous milk fat to study its thermal and structural properties. (Heating is always $2^{\circ} \mathrm{C} / \mathrm{min}$.) Both isothermal treatments at $-8^{\circ} \mathrm{C}$ and $+4^{\circ} \mathrm{C}$ are indicated as horizontal dashed lines. 
Table 2. Summary of the structural and thermal characteristics observed for anhydrous milk fat by x-ray diffraction and differential scanning calorimetry (DSC) techniques after cooling at the different rates indicated and during the subsequent heating.

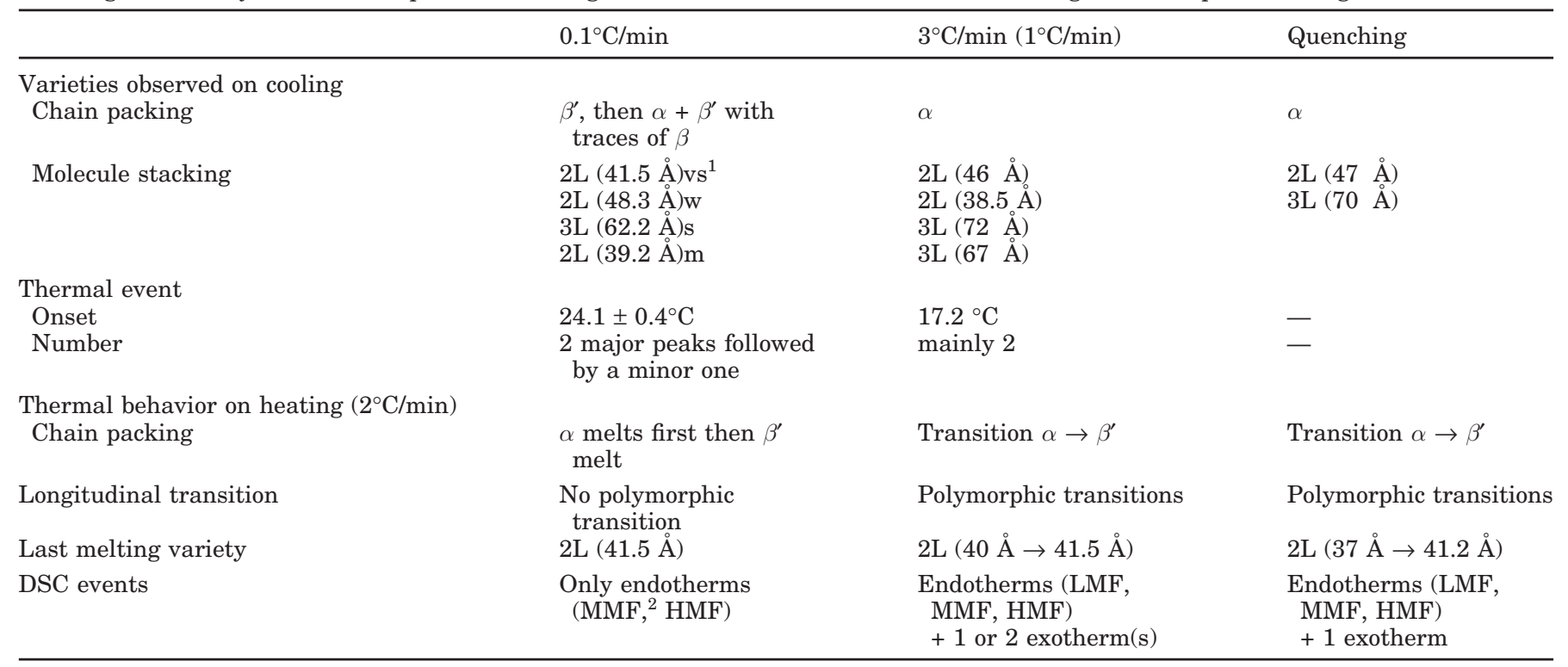

${ }^{1} \mathrm{vs}=$ Very strong, $\mathrm{s}=$ strong, $\mathrm{w}=$ weak, and $\mathrm{m}=$ medium.

${ }^{2} \mathrm{MMF}=$ Medium melting point fraction, $\mathrm{HMF}=$ high melting point fraction, and LMF = low melting point fraction.
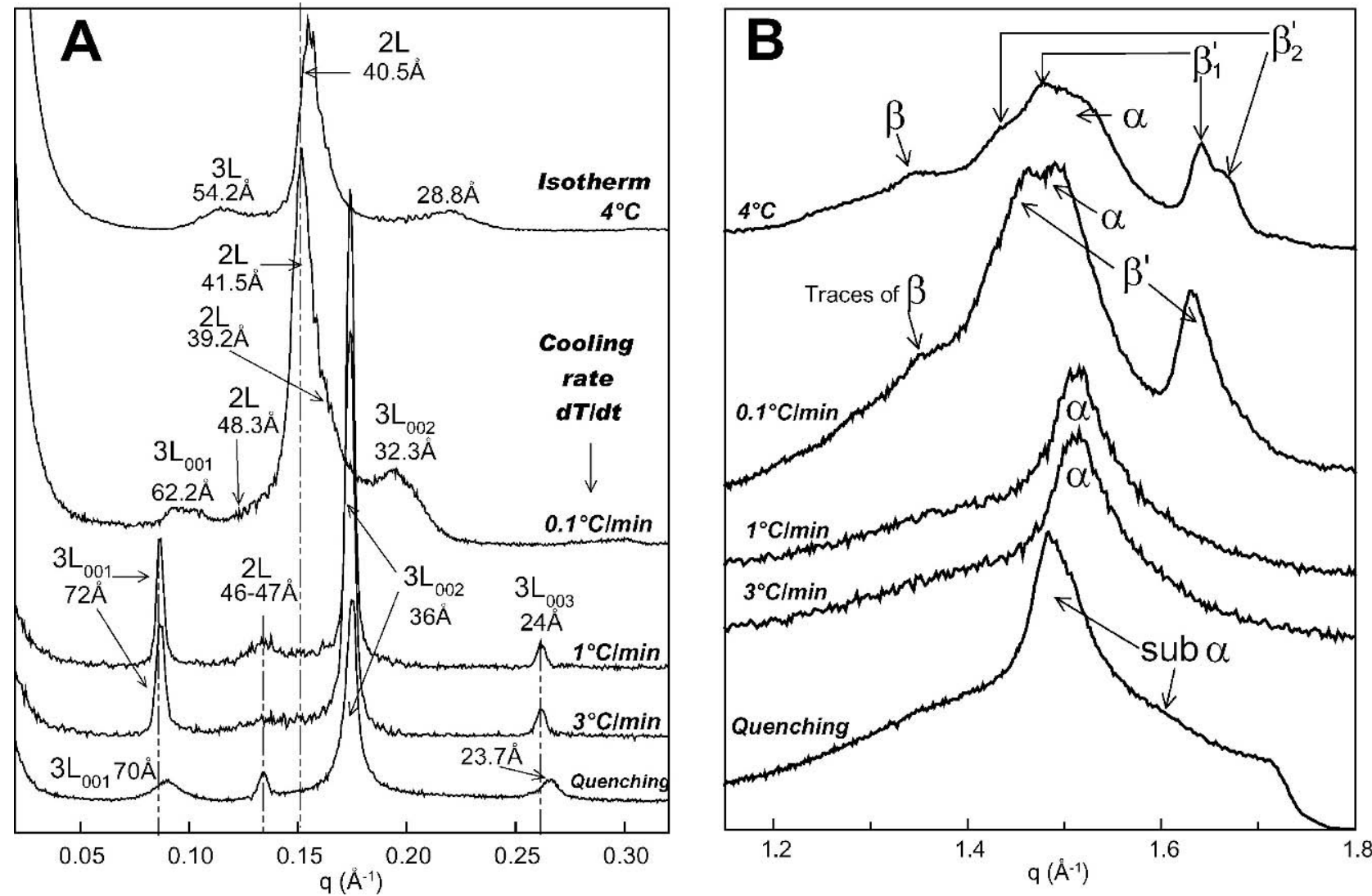

Figure 9. X-ray diffraction patterns recorded at $-8^{\circ} \mathrm{C}$ at A) small and B) wide angles after either cooling of anhydrous milk fat at different rates as indicated on the figure or isothermal conditioning at $4^{\circ} \mathrm{C}$ for $5 \mathrm{~d}$. $\mathrm{q}=$ Scattering vector, $2 \mathrm{~L}=$ bi-layered stacking, and $3 \mathrm{~L}=$ trilayered stacking 
fraction peak. This structure corresponds to the segregation of TG that forms crystals upon rapid cooling to $-8^{\circ} \mathrm{C}$. The TG composition of this structure is not known, but such a $2 \mathrm{~L}$ stacking may be formed by saturated TG with similar chain length (Small, 1986). The $3 \mathrm{~L}(70 \AA$ ) structure formed by quenching of AMF is characterized by a broad peak of diffraction that may be related to the formation of crystals of small sizes and/or to a longrange disorder in the longitudinal organization of the TG molecules in these lamellar structures, both effects being induced by rapid cooling. This peak broadening is partly related to crystal size reduction as observed by polarized light microscopy (see subsequent). However, it has been shown that the major origin of this peak broadening is the accumulation of structural defects and possibly related to interface curvature.

The broadening of the wide-angle diffraction peak recorded after quenching (sub- $\alpha$ form) corresponds to a mixture of crystals with the same polymorphic form but with different chemical compositions. Furthermore, the high value ( $4.22 \AA$ ) of the short spacing recorded likely corresponds to a more dense packing of the acylglycerol chains in the orthorhombic perpendicular subcell.

The lamellar structures formed by TG molecules on cooling at $3 \geq \mathrm{dT} / \mathrm{dt} \geq 1^{\circ} \mathrm{C} / \mathrm{min}$ are similar. They correspond to the coexistence of well-defined $3 \mathrm{~L}(72 \AA), 2 \mathrm{~L}$ (46 $\mathrm{A}$ ), and $2 \mathrm{~L}$ (38 to $39 \AA$ ) longitudinal stackings with a lateral organization of the chains in a hexagonal subcell.

Both $2 \mathrm{~L}$ and 3L packings being of $\alpha$ type, and long spacings ( 47 and $70 \AA$ ) being quite large, it is supposed that they correspond to chains perpendicular to the planes of the lamellae. On the contrary, smaller distances observed (41.5 and $62.2 \AA$ ) after slow cooling should correspond to tilted chains relative to these planes.

At slower cooling rates, $\mathrm{dT} / \mathrm{dt}=0.1^{\circ} \mathrm{C} / \mathrm{min}$, the structural behavior of AMF mainly corresponds to a crystallization in the $\beta^{\prime}$ form at about $24^{\circ} \mathrm{C}$ with the formation of a $2 \mathrm{~L}(41.5 \AA)$ longitudinal stacking, in coexistence mainly with a $\alpha 3 \mathrm{~L}(62.2 \AA)$ structure formed at about $13^{\circ} \mathrm{C}$. Moreover, traces of $\beta$ form were recorded (Figure 9B). For comparison, the XRD pattern recorded after storage of $\mathrm{AMF}$ at $4^{\circ} \mathrm{C}$ for $\mathrm{t}>100 \mathrm{~h}$ is presented on Figure 9B. These results show that the most stable longitudinal stackings of AMF TG that we observed correspond mainly to a $2 \mathrm{~L}$ stacking in coexistence with a $3 \mathrm{~L}$ organization of TG molecules. The differences in the thicknesses of the lamellar structures observed after cooling at $0.1^{\circ} \mathrm{C} / \mathrm{min}$ or storage at $4^{\circ} \mathrm{C}$ may be related to a tilt of the TG molecules in the lamellae, although a 54- $\AA$ period corresponds to a tilt (16 to $18 \AA$ ) reduction in thickness too large to correspond to single TG structure.

Whatever the cooling rate applied to AMF, initial crystallization occurs with a $2 \mathrm{~L}$ longitudinal stacking likely formed by trisaturated high melting point TG. As a function of the cooling rate, the time given to TG molecules to crystallize and incorporate $2 \mathrm{~L}$ lamellar structures is sufficient $\left(\leq 0.1^{\circ} \mathrm{C} / \mathrm{min}\right)$ or not $\left(\geq 1^{\circ} \mathrm{C} / \mathrm{min}\right)$ to achieve completion. When the slow-growing $2 \mathrm{~L}\left(\beta^{\prime}\right.$ and also $\alpha$ to a certain extent) structures have not completed, the fastgrowing 3L $\alpha$ structures develop. Both processes compete with each other for the incorporation of the same TG. However, both types of organization lead to mixed crystals constituted by TG with different chemical characteristics. Consequently, on heating, the evolution as a function of $\mathrm{T}$ of the $3 \mathrm{~L}$ structures leads to a polymorphic transition $3 \mathrm{~L} \rightarrow 2 \mathrm{~L}$. This transition 1 ) shows that structural rearrangements occur in the crystals to increase their thermodynamic stability and 2) confirms the existence of mixed crystals in the stable forms of AMF.

It is worth noting that the TG organization in these mixed crystals depends on the crystalline form with different accommodations of the structural parameters. In unstable forms, the broad and unique peak observed at wide angles $(\alpha)$ indicate a poor lateral organization that is compensated by very sharp small angle peaks related to a well-defined (3L) longitudinal organization. The reverse process is observed for stable varieties for which a more dense lateral packing attested by numerous wideangle sharp peaks is compensated by broadening of small angle peak (2L Figure $9 \mathrm{~A} ; 0.1^{\circ} \mathrm{C} / \mathrm{min}$ and $4^{\circ} \mathrm{C}$ ).

However, it is also worth noting that, contrary to what is observed for the lateral packing for which the subcell compactness defines a scale of stability, the stacking type has no relationship to stability. The $2 \mathrm{~L}$ stacking is not necessarily more, or less, stable than 3L one. For $\mathrm{AMF}$, the $2 \mathrm{~L}$ stacking observed previously at 41 to 42 $\AA$ is more stable than the $3 \mathrm{~L}$ one, but for cocoa butter, the $2 \mathrm{~L}$ is less stable than the $3 \mathrm{~L}$. Moreover, in AMF, the $2 \mathrm{~L}(47 \AA)$ that rapidly disappears as a function of time after quenching is even less stable than the $3 \mathrm{~L}$. However, the decrease of the longitudinal spacing associated with the tilt of the chain (when the vertical chain becomes tilted) is associated with an increase in stability.

Supramolecular behavior. Crystallization of AMF was monitored by polarized light microscopy. Figure 10 shows the crystals of AMF at $-8^{\circ} \mathrm{C}$ after cooling at different rates. Rapid cooling gives a large number of small crystals, and slow cooling provides large spherulitic particules: the faster the cooling, the smaller the crystals. When there are only weak differences observed at the molecular scale by XRDT between crystal obtained at -1 and $-3^{\circ} \mathrm{C} / \mathrm{min}$, a crystal mean size reduction is easily observed using polarized light microscopy.

However, a more careful analysis of the small angle $\mathrm{x}$-ray scattering data is presented in Figure 11. The contour plots of the scattering intensities observed at very small angles are drawn as a function of $\mathrm{T}$ and scattering 

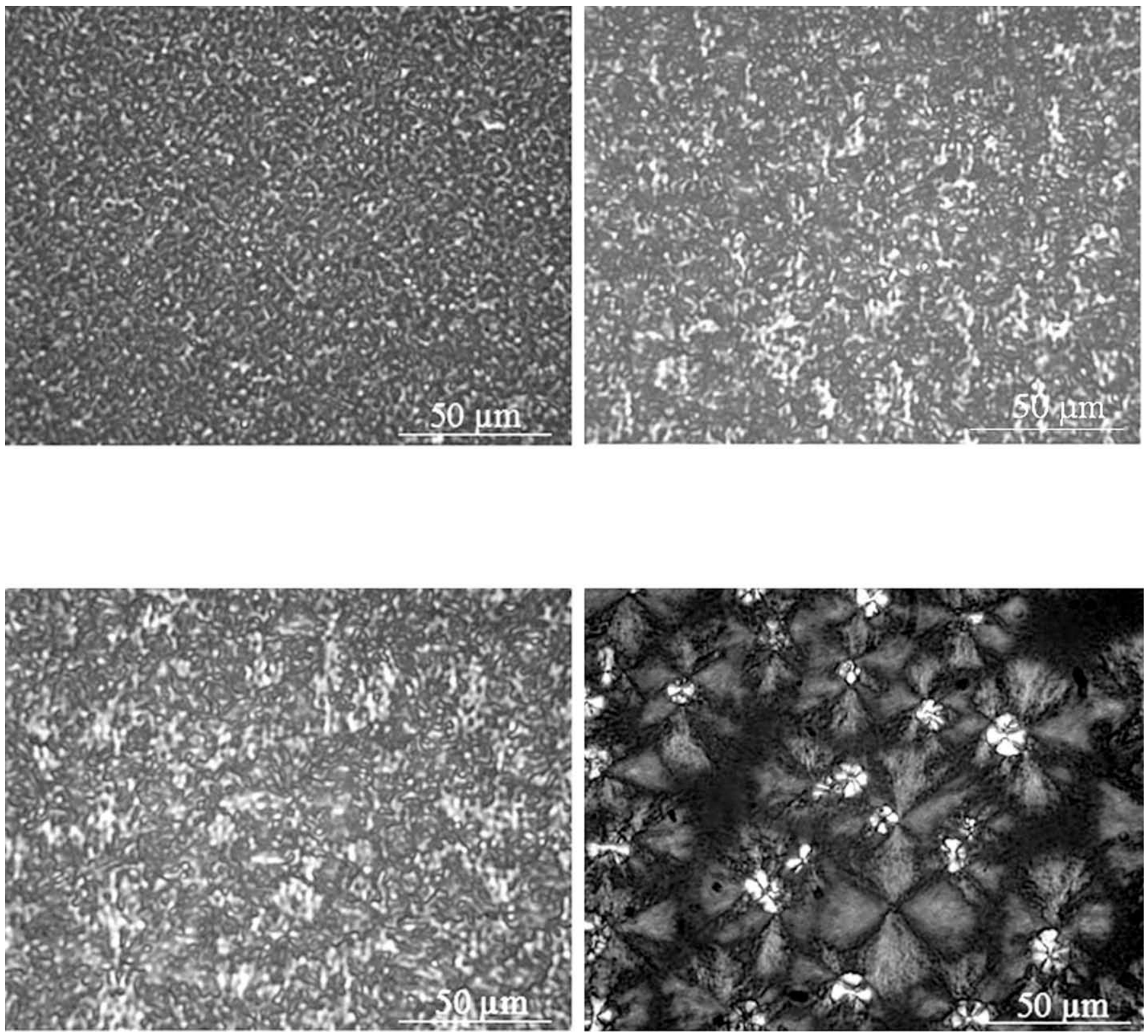

Figure 10. Polarized light microscopy pictures taken at $-8^{\circ} \mathrm{C}$ after quenching from $60^{\circ} \mathrm{C}$ (upper left) and cooling at $3^{\circ} \mathrm{C} / \mathrm{min}$ (upper right), $1^{\circ} \mathrm{C} / \mathrm{min}$ (lower left), and $0.2^{\circ} \mathrm{C} / \mathrm{min}$ (lower right).

vector for cooling at $3^{\circ} \mathrm{C} / \mathrm{min}$ (and partly for $1^{\circ} \mathrm{C} / \mathrm{min}$ ), and the subsequent heating is shown in Figure 6. Contour plots observed at very small angles are sensitive to crystal formation as well as to transition through the number and size of the crystals that depend on the cooling rate. These changes in scattering at very small angles correlate with the decrease in size observed by microscopy as a function of cooling rate (Figure 10). Such variations could be used as complementary measurements for crystal growth studies (Campos et al., 2002). This analysis illustrates the richness of small angle x-ray patterns. Each x-ray pattern recorded from long-chain compounds contains at least triple information about crystal type identification, quantification, and size.

\section{CONCLUSION}

Recently, the development of x-ray high-flux sources (synchrotron) has permitted 1) the study of the polymorphism and phase transitions displayed by complex TG mixtures as a function of $\mathrm{T}$ and 2) a comparison of these data with DSC recordings. However, independent of the 


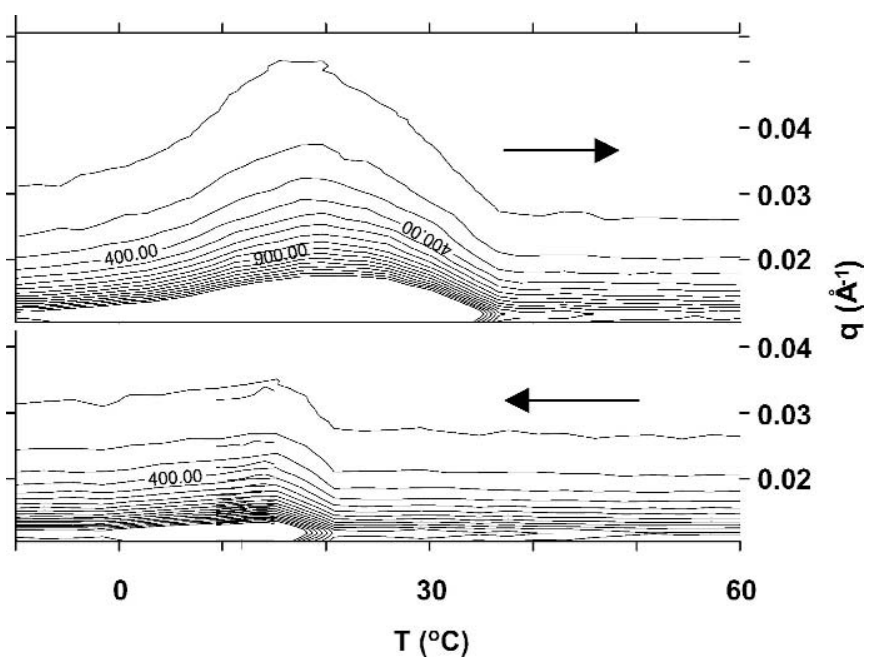

Figure 11. Contour plots of the scattering observed at very small angles. Equidistant contour levels (intensities corresponding to levels 400 and 900 are indicated), corresponding to 3-D plots shown Figure 6 , are drawn as a function of temperature $(\mathrm{T})$ and scattering vector q. Bottom: cooling at $3^{\circ} \mathrm{C} / \mathrm{min}$; top: heating at $2^{\circ} \mathrm{C} / \mathrm{min}$ (part of the contour plot observed on cooling at $1{ }^{\circ} \mathrm{C} / \mathrm{min}$ is shown as a superimposition in the cooling part in the T range from about 15 to $10^{\circ} \mathrm{C}$ for comparison.

cooling rate, the coexistence of several crystalline organizations is frequently observed at both small and wide angles. Thus, the challenge is then to relate the longitudinal stacking and lateral packing associated with the same crystalline variety. In this series of studies on milk fat polymorphism, the careful analysis of peak intensities and positions as a function of $\mathrm{T}$ or time allowed intermediate complexity cases to solve the establishment of such relationship; this was not yet possible in the most complex cases. In this respect, it is worth noting that, thanks to the resolution obtained with the various setups used in these studies, the small-angle analysis performed for the first time for milk fat is essential in such an identification of the thermally induced processes.

The experiments reported in this study give deeper insight into the crystallization and polymorphic transitions of milk fat. By cooling milk fat at different cooling rates, the phase behavior was investigated. It was found that the TG of milk fat can crystallize in 4 different polymorphic subcell types: sub- $\alpha, \alpha, \beta^{\prime}$, and $\beta$ with reversible $\alpha \rightarrow$ sub- $\alpha$ and irreversible $\alpha \rightarrow \beta^{\prime} \rightarrow \beta$ transition, and corresponding $2 \mathrm{~L}$ and $3 \mathrm{~L}$ stackings. This last stacking is characteristic of the longitudinal arrangement of TG in which short or unsaturated fatty acid chains are mixed together with long saturated fatty acid chains.

The existence of such a polymorphism may have an important consequence in rheological behavior. Indeed, the crystal polymorph has primordial importance for final product consistency and acceptability. Smaller crys- tals lead to firmer fat products, whereas larger crystals give a sensation of sandiness in the mouth.

\section{ACKNOWLEDGMENTS}

The authors thank ARILAIT Recherches (Paris, France) and ANRT (Paris, France) for supporting this research. They also thank La Laiterie du Val d'Ancenis (Ancenis, France) for supplying cream samples from which AMF was extracted. They also thank A. Riaublanc and S. Bernadou as well as all the members of the Steering Committee for Fat Programs of ARILAIT Recherches for help in this study at various steps.

\section{REFERENCES}

Blanton, T. N., C. L. Barnes, and M. Lelental. 2000. Preparation of silver behenate coatings to provide low-to mid-angle diffraction calibration. J. Appl. Crystallogr. 33:172-173.

Campos, R., S. S. Narine, and A. G. Marangoni. 2002. Effect of cooling rate on the structure and mechanical properties of milk fat and lard. Food Res. Int. 35:971-981.

Christie, W. W. 1995. Composition and structure of milk lipids. Pages 1-28 in Advanced Dairy Chemistry, Vol. 2, Lipids, 2nd ed. P. F. Fox, ed. Chapman \& Hall, London, UK.

deMan, J. M. 1961. Physical properties of milk fat. II. Some factors influencing crystallization. J. Dairy Res. 28:117-122.

Hagemann, J. W. 1988. Thermal behavior and polymorphism of acylglycerides. Pages 9-95 in Crystallization and Polymorphism of Fats and Fatty Acids. N. Garti and K. Sato, ed. Marcel Dekker, Inc., New York, NY.

Keller, G., F. Lavigne, L. Forte, K. Andrieux, M. Dahim, C. Loisel, M. Ollivon, C. Bourgaux, and P. Lesieur. 1998. DSC and X-Ray diffraction coupling. Specifications and applications. J. Thermal Anal. 51:783-791.

Keller, G., F. Lavigne, C. Loisel, M. Ollivon, and C. Bourgaux. 1996. Investigation of the complex thermal behavior of fats: Combined DSC and X-ray diffraction techniques. J. Thermal Anal. 47:1545-1565.

Larsson, K. 1972. Molecular arrangement in glycerides. Fette Seifen Anstrichm. 74:136-142.

Lavigne, F. 1995. Polymorphisme et transitions de phases des triglycèrides. Applications aux propriétés thermiques et structurales de la matière grasse laitière anhydre et de ses fractions. $\mathrm{Ph}$. D. Univ. Paris VII, Paris XI et E.N.S.I.A., France.

Lavigne, F., C. Bourgaux, and M. Ollivon. 1993. Phase transition of saturated triglycerides. suppl. J. Phys. I. 3:137-140.

Loisel, C., G. Keller, G. Lecq, C. Bourgaux, and M. Ollivon. 1998. Phase transition and polymorphism of cocoa butter. J. AOCS 75:425-439.

Lopez, C., C. Bourgaux, P. Lesieur, S. Bernadou, G. Keller, and M. Ollivon. 2001a. Thermal and structural behavior of milk fat. 3 . Influence of cooling rate and droplet size on cream crystallization. J. Colloid Interface Sci. 254:64-78.

Lopez, C., F. Lavigne, P. Lesieur, C. Bourgaux, and M. Ollivon. 2001b. Thermal and structural behavior of milk fat. 1.Unstable species of anhydrous milk fat. J. Dairy Sci. 84:756-766.

Lopez, C., F. Lavigne, P. Lesieur, G. Keller, and M. Ollivon. 2001c. Thermal and structural behavior of anhydrous milk fat. 2.Crystalline forms obtained by slow cooling. J. Dairy Sci. 84:2402-2412.

Lopez, C., P. Lesieur, C. Bourgaux, G. Keller, and M. Ollivon. 2001d. Thermal and structural behavior of milk fat. 2. Crystalline forms obtained by slow cooling of cream. J. Colloid Interface Sci. 240:150-161.

Marangoni, A. G., and R. W. Lencki. 1998. Ternary phase behavior of milk fat fractions. J. Agric. Food Chem. 46:3879-3884.

Mulder, H. 1953. Melting and solidification of milk fat. Neth. Milk Dairy J. 7:149-174. 
Mulder, H., and P. Walstra. 1974. The Milk Fat Globule. Center for Agricultural Publishing and Documentation, Wageningen, The Netherlands.

Ollivon, M. 1982. Contribution à l'étude des triglycérides: Polymorphisme et transitions de phase. Ph.D. dissertation, Univ. Pierre et Marie Curie, Paris, France.

Ollivon, M., and R. Perron. 1992. Manuel des Corps Gras. A. Karleskind and J. P. Wolff, ed. Lavoisier, Paris, France.

Schaap, J. W., H. T. Badings, D. G. Schmidt, and E. Frede. 1975. Differences in butterfat crystals crystallized from acetone and from the melt. Neth. Milk Dairy J. 29:242-252.

Small, D. M. 1986. Handbook of Lipid Research. The Physical Chemistry of Lipids. From Alkanes to Phospholipids. Plenum Press, New York, NY. ten Grotenhuis, E., G. A. van Aken, K. F. van Malssen, and H. Schenk. 1999. Polymorphism of milk fat studied by differential scanning calorimetry and real-time X-ray powder diffraction. J. AOCS 76:1031-1039.

Timms, R. E. 1979. The physical properties of blends of milk fat with beef tallow and beef tallow fractions. Aust. J. Dairy Technol. 34:60-65.

Timms, R. E. 1980. The phase behaviour and polymorphism of milk fat, milk fat fractions and fully hardened milk fat. Aust. J. Dairy Technol. 35:47-53.

van Beresteyn, E. C. H. 1972. Polymorphism in milk fat in relation to the solid/liquid ratio. Neth. Milk Dairy J. 26:117-130.

Woodrow, I. L., and J. M. deMan. 1968. Polymorphism in milk fat shown by x-ray diffraction and infrared spectroscopy. J. Dairy Sci. 51:996-1000. 